\section{Cre-loxP recombination vectors for the expression of Riken Arabidopsis full-length cDNAs in plants}

Toshiro Shigaki ${ }^{1}$, Mamata Kole ${ }^{1}$, John M. Ward ${ }^{2}$, Heven Sze ${ }^{3}$, and Kendal D. Hirschi ${ }^{1}$

${ }^{1}$ Baylor College of Medicine, Houston, TX, ${ }^{2}$ University of Minnesota, St. Paul, MN, and ${ }^{3}$ University of Maryland, College Park, MD, USA

BioTechniques 39:301 - 303 (September 2005)
An essential and often arduous task in plant functional genomic studies is the expression of full-length cDNAs in plants. Historically, this step has been confounded by technical hurdles, including obtaining full-length cDNAs and cloning these cDNAs into frequently cumbersome plant expression vectors. The Arabidopsis full-length cDNAs available from Riken (RAFL clones; Riken, Saitama, Japan) are now an invaluable resource for functional studies (1). To date, these inserts have often been engineered into large binary vectors by traditional restriction digestion and ligation. To overcome this limitation, we have developed a system that can streamline the expression of RAFL cDNAs in plants.

The RAFL cDNAs are cloned in modified pBlueScript $^{\circledR}$ vectors (Stratagene, La Jolla, CA, USA), and the insert must be digested with SfiI, as it is the only available restriction site for the transfer. Most binary vectors currently in use do not have SfiI in the multiple cloning site. Therefore a cloning "detour" is required; namely, gene-specific restriction sites must be added at the $5^{\prime}$ and $3^{\prime}$ ends of the cDNA by PCR.

Binary vectors are often large, and ligation into these vectors is technically more difficult than cloning into small vectors. Recently, recombination based cloning systems that can forgo restriction digestion and ligation have been developed (2-4). These systems are highly precise and efficient. Currently, publicly available plant expression vectors with recombination functionality are based on the Gateway ${ }^{\circledR}$ system (Invit- rogen, Carlsbad, CA, USA); however, the in vitro recombination reactions require a commercial enzyme mixture, making the cloning less cost-effective. Nonetheless, numerous Gatewayadapted vectors are freely distributed $(3,4)$.

Our system is an alternative to PCR-based systems, which sometimes introduce errors and require resequencing of the cDNA clones. Our system can streamline the expression of RAFL cDNAs in plants using a small vector with RAFL-compatible $S f i$ sites and the publicly available Cre-loxP site-specific recombination system. Cre-loxP recombination was discovered in the bacteriophage P1 (5) and takes place between two 34bp loxP sequences in which the Cre recombinase protein binds. In the Cre-loxP system, a gene of interest is first cloned in a small vector called a univector that contains a conditional replication origin $\gamma$ from Escherichia coli plasmid R6K (2). The replication of univectors requires pi protein encoded by the pir gene. Therefore, the vectors must be transformed in an $E$. coli strain expressing pi protein, such as BW23474. The vector cannot multiply in common laboratory strains DH5 $\alpha$ or TOP10. After the recombination with
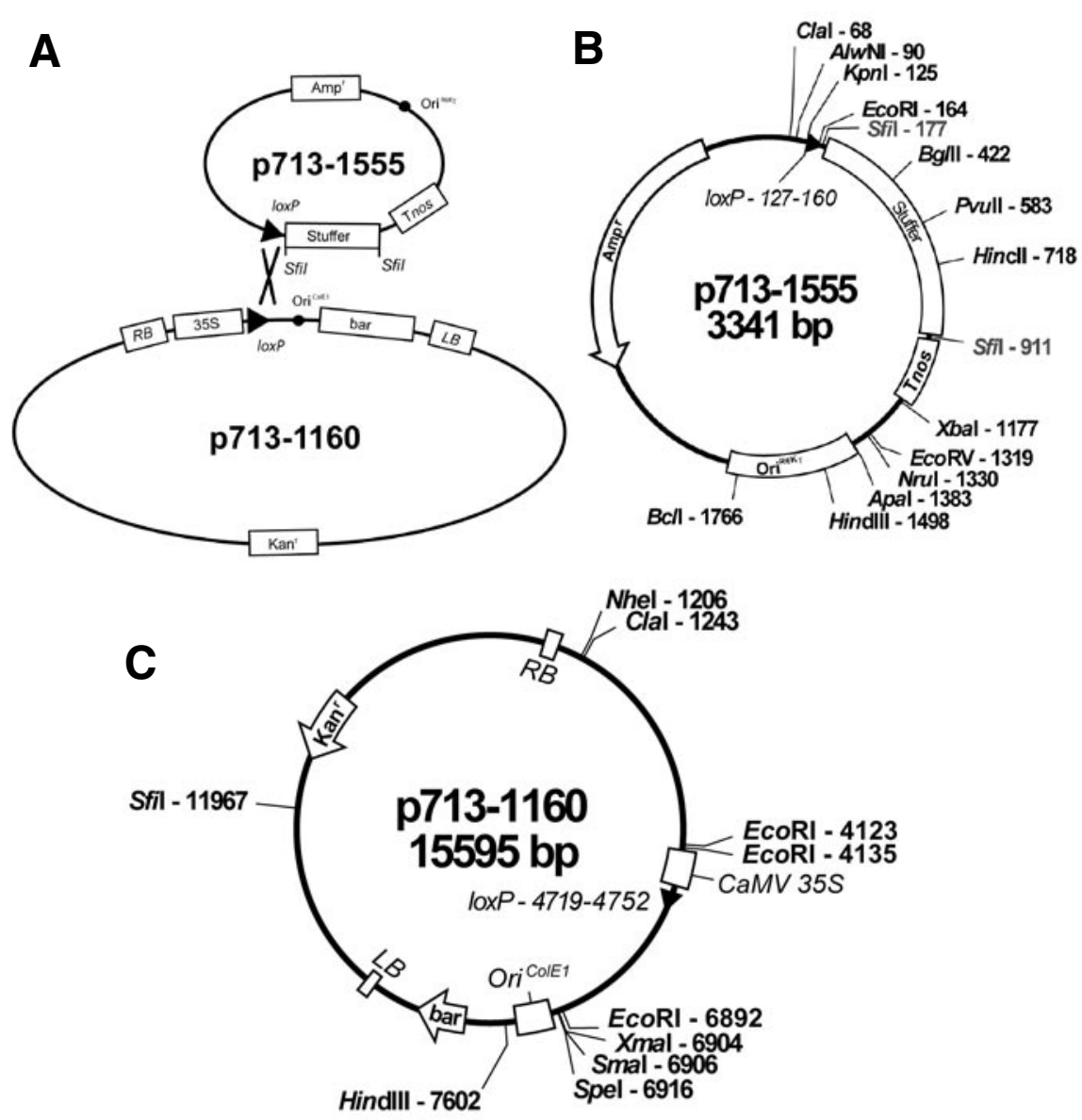

Figure 1. The Cre-loxP recombination system for gene expression in plants. (A) Representation of the Cre-loxP recombination for plant expression. (B) Physical map of the univector p713-1555. (C) Physical map of the host vector p713-1160.

BioTechniques 301 
a host vector, which has a ColE1 replication origin and confers the ability to transform destination cells, the fused plasmid can be selected by simply transforming into ColE1-compatible strains (e.g., DH5 $\alpha$ or TOP10). This efficient and precise approach has been successfully used to make expression vectors for mammals, insects, bacteria, and yeast (2). Recently, we developed a reporter gene system that utilizes CreloxP recombination for plant promoter activity analysis (6). We report here a new Cre-loxP recombination vector system for gene expression in plants.

The univector for the plant expression system was constructed by inserting a 721-bp stuffer fragment from the Arabidopsis cation exchanger $C A X 2$ gene (At3g13320) and the 255 bp terminator ( $3^{\prime}$ untranslated region) from the Agrobacterium tumefaciens nopaline synthase (nos) gene between the EcoRI and $X b a \mathrm{I}$ sites of pUNI(Amp)-DBD $\left(\right.$ GenBank $^{\circledR}$ accession no. AF149266). SfiI sites were added to the $5^{\prime}$ and $3^{\prime}$ ends of the stuffer for cloning RAFL cDNAs. SfiI creates unique sitespecific three-base overhangs upon digestion. The overhang sequences in the new univector were made so that they are compatible with RAFL clones; namely, the $S f i$ sequence proximal to the loxP is $5^{\prime}$-GGCCAAATCGGCC-3' (SfiI-aat), and SfiI sequence distal to the $\operatorname{lox} P$ is $5^{\prime}$-GGCCATAAGGGCC-3'

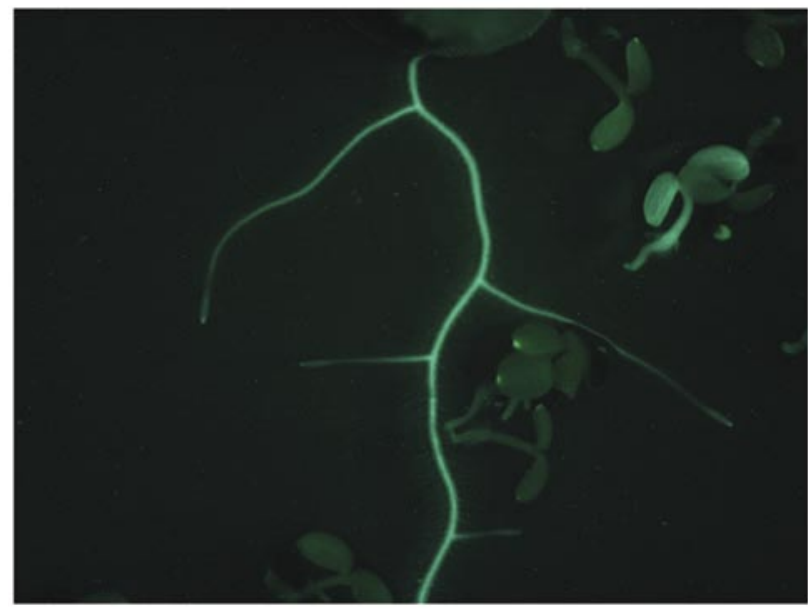

Figure 2. A transgenic Arabidopsis seedling expressing the modified green fluorescent protein (smGFP) construct made by the Cre-loxP recombination system. The picture was taken with Nikon SMZ-1500 fluorescent microscope (Nikon, Tokyo, Japan), using the X-Cite ${ }^{\mathrm{TM}} 120$ PC Fluorescence Illumination System (EXFO Life Sciences Group, Mississauga, ON, Canada) with excitation wavelength $470 / 40 \mathrm{~nm}$ and emission wavelength $525 / 50 \mathrm{~nm}$.
(SfiI-taa). This vector has an ampicillin resistance marker for selection in the $E$. coli strain BW23474. The new vector has been termed p713-1555 (GenBank accession no. DQ062657) (Figure 1, A and B).

The host vector was constructed by inserting a cauliflower mosaic virus $35 \mathrm{~S}$ promoter fragment in the KpnI site of p713-905 (6) (GenBank accession no. AY860533). Immediately downstream of the $35 \mathrm{~S}$ promoter is a $\operatorname{lox} P$ sequence used for Cre recombinase-mediated recombination. This vector has a kanamycin selection marker for bacteria and a glufosinateammonium (BASTA) selection marker for plants. This new vector has been termed p713-1160 (GenBank accession no. DQ062658) (Figure 1, A and C).

In order to easily monitor the efficacy of our system, we have tested the expression of a modified green fluorescent protein (smGFP) (7) in Arabidopsis. This gene is obviously not a RAFL clone, and accordingly, we have added the RAFL clone-compatible $S f i$ sites by PCR to the coding sequence of smGFP for cloning into p713-1555.

The in vitro recombination between the resultant univector clone with smGFP and the host vector p713-1660 was performed as described by Liu et al. (2) (Figure 1A). Briefly, a reaction mixture consisting of $1 \mu \mathrm{L}(100 \mathrm{ng})$ univector plasmid, $1 \mu \mathrm{L}$ (100 ng) host vector plasmid,

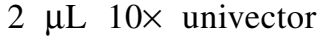
plasmid-fusion system (UPS) buffer [1× buffer consisting of $50 \mathrm{mM}$ Tris- $\mathrm{HCl}, \mathrm{pH}$ $7.5,10 \mathrm{mM} \mathrm{MgCl}_{2}$, $30 \mathrm{mM} \mathrm{NaCl}$, and 0.1 $\mathrm{mg} / \mathrm{mL}$ bovine serum albumin (BSA)], 15 $\mu \mathrm{L}$ water, and $1 \mu \mathrm{L}$ $(0.1-1.0 \quad \mu \mathrm{g}) \quad$ Cre protein was incubated at $37^{\circ} \mathrm{C}$ for $20 \mathrm{~min}$. Cre protein was produced according to the method of Liu et al. (2). Five microliters of the reaction was used to transform $E$. coli and was selected on media containing $100 \mu \mathrm{g} / \mathrm{mL}$ ampicillin and $50 \mu \mathrm{g} / \mathrm{mL}$ kanamycin. Using this protocol, we typically obtain several hundred colonies.

To confirm the correct recombination, the recombined vectors were digested with EcoRI. The host vector p713-1160 has three EcoRI sites that produce two distinct bands (and one short band that is difficult to visualize) upon digestion. When recombined, the host vector acquired one EcoRI site from the univector p713-1555. We tested univector clones with various inserts to determine the recombination efficiency of the system. From a total of 32 examined, 29 (91\%) produced correct restriction patterns. The fusion junctions of five selected clones were sequenced, and all were found to be error-free.

After confirming the correct CreloxP recombination had taken place, the recombined vector was transformed into Agrobacterium tumefaciens strain GV3101 by the freeze-thaw method (8) and selected on YM media (6) with $10 \mu \mathrm{g} / \mathrm{mL}$ gentamycin and $50 \mu \mathrm{g} / \mathrm{mL}$ kanamycin. The Agrobacterium was used to transform Arabidopsis by the floral dip method (9). T1 seeds were selected on half-strength MurashigeSkoog media (10) containing BASTA at a concentration of $20 \mu \mathrm{g} / \mathrm{mL}$. Tenday-old T1 transformants were excited with blue light and observed for the production of green fluorescence from ectopically expressed smGFP. All transformants examined showed fluorescence in the roots, demonstrating the utility of the Cre-loxP vector (Figure 2).

Our host vector p713-1160 is also compatible with the fully sequenced Arabidopsis open reading frame (ORF) clones available from the Arabidopsis Biological Resource Center (11). These ORF clones are especially useful when the proteins are expressed in heterologous systems.

This expression system has two distinct advantages, similar to the approach used to generate myc tags of Arabidopsis ORF in planta (11). First, the univector has the SfiI restriction sites that are compatible with RAFL cDNAs making the cloning step routine and independent of the cDNA sequence. Specifically, due to the small size of the vector, cloning is straight- 
forward. Each SfiI site generates sitespecific 3-bp overhangs upon digestion. Thus, the fragments can only ligate back to restore the original sequence (12). Therefore, internal SfiI sites within the cDNAs do not compromise the ligation step. Second, the Cre-loxP recombination is easy, economical, fast, accurate, and efficient. While the wide array of Gateway vectors have many of these advantages, they must be recombined with a proprietary enzyme mixture. In contrast, the Cre recombinase can be made inexpensively in any laboratory. The vectors and $E$. coli strains described in this paper are available upon request for noncommercial applications. In summary, our new vector set is a valuable tool in the analysis of plant gene function.

\section{ACKNOWLEDGMENTS}

We are grateful to Stephen J. Elledge, Bonnie Bartel, and Sherry LeClere for the template vectors. We also thank Ian Rees for the artwork. This study was funded by the National Science Foundation grant nos. 0209777 to K.H., 0209792 to J.W., and 0209788 to H.S.

\section{COMPETING INTERESTS STATEMENT}

The authors declare no competing interests.

\section{REFERENCES}

1. Seki, M., M. Narusaka, A. Kamiya, J. Ishida, M. Satou, T. Sakurai, M. Nakajima, A. Enju, et al. 2002. Functional annotation of a full-length Arabidopsis cDNA collection. Science 296:141-145.

2.Liu, Q., M.Z. Li, D. Leibham, D. Cortez, and S.J. Elledge. 1998. The univector plasmid-fusion system, a method for rapid construction of recombinant DNA without restriction enzymes. Curr. Biol. 8:1300-1309.

3. Karimi, M., D. Inzé, and A. Depicker. 2002. GATEWAY vectors for Agrobacterium-mediated plant transformation. Trends Plant Sci. 7:193-195.

4. Curtis, M.D. and U. Grossniklaus. 2003. A Gateway cloning vector set for high-throughput functional analysis of genes in planta. Plant Physiol. 133:462-469.

5. Abremski, K., R. Hoess, and N. Sternberg. 1983. Studies on the properties of P1 site-spe- cific recombination: evidence for topologically unlinked products following recombination. Cell 32:1301-1311.

6. Shigaki, T., R. Ravindranadha, A.B. Sivitz, J.M. Ward, H. Sze, and K.D. Hirschi. 2005. The Cre-loxP recombination-based reporter system for plant transcriptional expression studies. Plant Mol. Biol. 58:65-73.

7. Davis, S.J. and R.D. Vierstra. 1998. Soluble, highly fluorescent variants of green fluorescent protein (GFP) for use in higher plants. Plant Mol. Biol. 36:521-528.

8. An, G., P.R. Ebert, A. Mitra, and S.B. Ha. 1988. Binary vectors, p. 1-19. In S.B. Gelvin and R.A. Schilperoort (Eds.), Plant Molecular Biology Manual A3. Kluwer, Dordrecht.

9. Clough, S.J. and A.F. Bent. 1998. Floral dip: a simplified method for Agrobacterium-mediated transformation of Arabidopsis thaliana. Plant J. 16:735-743.

10.Murashige, T. and F. Skoog. 1962. A revised medium for rapid growth and bioassays with tobacco tissue cultures. Physiol. Plant. 15:473-497.

11.Yamada, K., J. Lim, J.M. Dale, H. Chen, P. Shinn, C.J. Palm, A.M. Southwick, H.C. Wu, et al. 2003. Empirical analysis of transcriptional activity in the Arabidopsis genome. Science 302:842-846.

12.Shigaki, T. and K.D. Hirschi. 2002. Chimeric gene construction without reference to restriction sites. BioTechniques 32:736-740.

Received 25 May 2005; accepted 29 June 2005.

Address correspondence to Kendal D. Hirschi, Children's Nutrition Research Center, Baylor College of Medicine, 1100 Bates St., Houston, TX 77030, USA. e-mail: kendalh@bcm.tmc.edu

To purchase reprints

of this article, contact

apfeffer@BioTechniques.com 